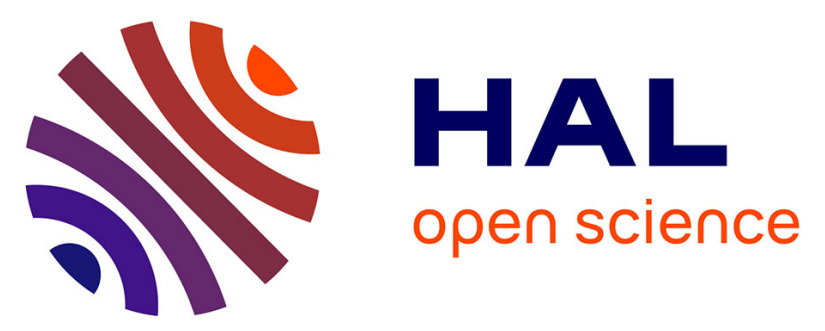

\title{
Auction Mechanisms for Management of Steam generated by High Temperature Reactors
}

Blażej Chmielarz, Cédric Herpson, Alexandre Bredimas, Zahia Guessoum

\section{To cite this version:}

Blażej Chmielarz, Cédric Herpson, Alexandre Bredimas, Zahia Guessoum. Auction Mechanisms for Management of Steam generated by High Temperature Reactors. International Conference on Practical Applications of Agents and Multi-Agent Systems (PAAMS), Frank Dignum - Umeå University (Sweden), Oct 2021, Salamanca, Spain. pp.64-76, 10.1007/978-3-030-85739-4_6 . hal-03283597

\section{HAL Id: hal-03283597 https://hal.science/hal-03283597}

Submitted on 12 Jul 2021

HAL is a multi-disciplinary open access archive for the deposit and dissemination of scientific research documents, whether they are published or not. The documents may come from teaching and research institutions in France or abroad, or from public or private research centers.
L'archive ouverte pluridisciplinaire HAL, est destinée au dépôt et à la diffusion de documents scientifiques de niveau recherche, publiés ou non, émanant des établissements d'enseignement et de recherche français ou étrangers, des laboratoires publics ou privés.

\section{()ㅜ) $\Theta$}

Distributed under a Creative Commons Attribution - NoDerivatives| 4.0 International 


\title{
Auction Mechanisms for Management of Steam generated by High Temperature Reactors
}

\author{
Błażej Chmielarz ${ }^{1,2}$, Cédric Herpson ${ }^{2}$, Alexandre Bredimas ${ }^{1}$, Zahia Guessoum ${ }^{2,3}$ \\ 1 USNC Europe, 91190 Gif-sur-Yvette, France \\ 2 Sorbonne Université, CNRS, LIP6, F-75005 Paris, France \\ ${ }^{3}$ CReSTIC, EA 3804, URCA, F-5100 Reims, France
}

\begin{abstract}
Steam networks in existing chemical facilities are typically highly centralized and operate on long-term contracts. It results in energy systems with overcapacity of production, exceeding the demand several times. Facilities which remain idle or utilize fuel sub-optimally create unnecessary costs and possess high carbon footprint. To optimize fuel consumption, increase profits and lower emissions, we propose to decentralize and trade steam distribution with energy producers, chemical facilities and energy storage constituting autonomous agents. In this paper we describe a model of short-term trading of steam through multi-stage auction mechanisms. The goal of this model is to sell all produced steam every timestep while meeting the demand and including both smaller and larger parties in the negotiations fairly.
\end{abstract}

Keywords: Energy, Nuclear, CHP, VPP, Steam, Multi-agent, auction

\section{Introduction}

Steam is a common energy carrier which industry complexes require an uninterrupted flow of to operate correctly. In some cases, live steam is used in chemical processes [1]. The demand of each consumer of steam in real systems is typically not a constant. Failing to meet the demand results in pressure changes in the main steam loop, which, in real systems, could cause lower efficiency, or even outright cease, of processes in a facility of the end user. This level of importance often leads owners of the chemical complexes to overbuild their energy installations where energy production capacities exceed $200 \%$ of demand. The large installed redundancies are dictated by the probability of spikes in demand coinciding with maintenance or malfunctioning of multiple boilers. Various kinds of energy generating technologies can be found in the complexes. Different physical principles of operation among the technologies require trading mechanisms which can accommodate them fairly.

Steam users have highly specific contracts with steam producers which ensure steam delivery on demand. The contracts often span a year or more but they cannot accurately put a price for minute-to-minute costs of operation a year ahead due to highly varied demand throughout the year [2]. It leads to complicated annual renegotiations between producers and consumers based on past energy consumption and deviations from quotas. The potential impact of day-to-day decisions regarding current energy demand on future 
contracts cannot be fully understood at the moment of making said decisions. It can lead to economically and environmentally disadvantageous decisions of both consumers and producers of steam [3].

Considering how crucial steam is to the business of everyone in the system, the customers need to have more than one mean of establishing future supply and demand to better plan their operations. The current approach to meet the steam demand of chemical complexes works but is sub-optimal. Having multiple boilers on standby is costly, due to committed capital and recurring fixed costs, which need to be recuperated by the system. Addressing swift changes in demand could happen in a more cooperative fashion through market-driven approach - dynamic pricing is used to keep as many power plants as close to optimal operating conditions as possible.

The energy system proposed in this paper presents an alternate approach in which control of energy production is decentralised. The model trades steam in multi-stage auctions. The goal of the model is to sell all available steam every time-step while meeting the demand, including both smaller and larger parties in the negotiations fairly and lowering emissions without losing profits. Decentralisation of energy sales is achieved through agent-based modelling, where the agents, who represent energy producers, consumers or prosumers of the system, trade steam and electricity through auctions in two different time-frames: short- and long-term. High Temperature Reactors (HTRs) are added to the energy mix to assess their impact on emissions and economics.

The paper is organized as follows. Section 2 presents the entire system and the different types of agents and mechanisms. Section 3 describes the methodology of the short-term auctioning. Section 4 describes the setup of the test system and its implementation as well as the data that will be used for simulations. Section 5 presents a brief overview of the literature and highlights open issues before concluding.

\section{System overview and Agentification}

The proposed approach agentifies the entities that make up the Hybrid Cogeneration Plant ${ }^{4}$ into two main agent categories - concrete and special.

Physical components are represented by a set of discrete, concrete agents - boilers, nuclear reactors, energy storage, steam turbines, chemical plants or hydrogen production plants. The special agents are responsible for trade between concrete-agents, broadcasting external system variables or managing steam delivery (Fig. 1).

Each of the concrete agents contains a logical and a physical model. The system separates concrete agents at the common denominator of the physical system - steam. This separation facilitates arranging the concrete agents into Virtual Power Plants (VPPs). A VPP is any aggregate of concrete agents who will be represented as a singular trade participant (Fig. 2). Additionally, the high-level separation between agents permits the sale of energy in the system while allowing agents whose inner workings are black boxes, which is necessary in real-life energy systems where operational details are typically classified.

\footnotetext{
${ }^{4}$ A hybrid energy system is one where there is typically more than one product out of one or more energy energy sources, like co-generation of steam and electricity from both gas and coal boilers. It can also refer to more than one energy source producing the same ware.
} 


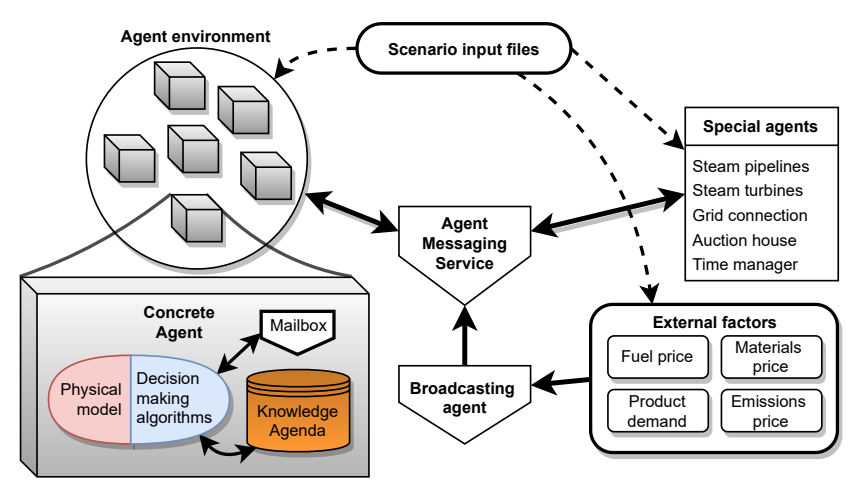

Fig. 1: Overview of the system

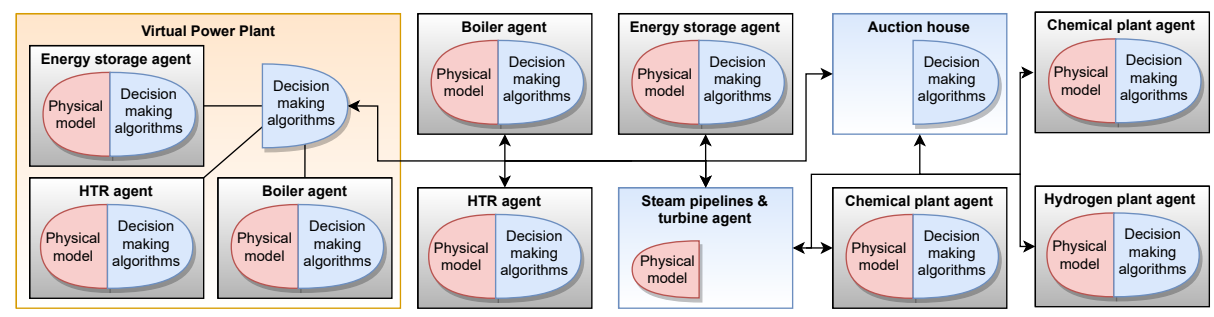

Fig. 2: Visualisation of aggregated agents and connections between them

Steam is a fungible good which should be sold in its entirety once it is produced in order to avoid upsetting the stability of the entire system. Separate auction mechanisms exist for long- and short-term trades. The short-term trade covers no more than a few minutes, trading steam produced inevitably in that short timeframe due to operational constraints of steam producers. The long-term sales offer steam futures - executable options to buy a certain amount of steam sometime in the future at a set price, and long-term contracts for steam at a constant volume and price. Contrary to short-term trades which are frequently suboptimal, long-term trades are well established processes not considered as an issue for the sector's players. We thus focus on the the former in this work.

Two auction systems will be presented in the paper - classic and reverse dutch auctions [4]. Classic auctions are ones issued by sellers. Reverse auctions are issued by buyers. Dutch auctions are more suited to sales of bulk goods, such as electricity, fuel or grains, whereas english auctions are better suited for single item sales [5]. The need to sell the entirety of produced steam in the short-term trade has led us to dutch-style auctions.

In summary, the system is designed to sell the entirety of its wares through auctions, with short-term auctions being either classic or reverse dutch auctions. Agents of the system can have logical and physical layers. Special agents lack either the physical or logical layer. Concrete agents contain both layers. They are capable of independent decision-making and can aggregate into VPPs and be represented as one trade participant. 


\section{Short-term auction mechanisms}

The short-term trade of steam is separated into three protocols that are described in the following sections: 1) Prediction of aggregated supply and demand of steam, 2) Two-phase auctioning of steam and 3) Execution of accepted trades.

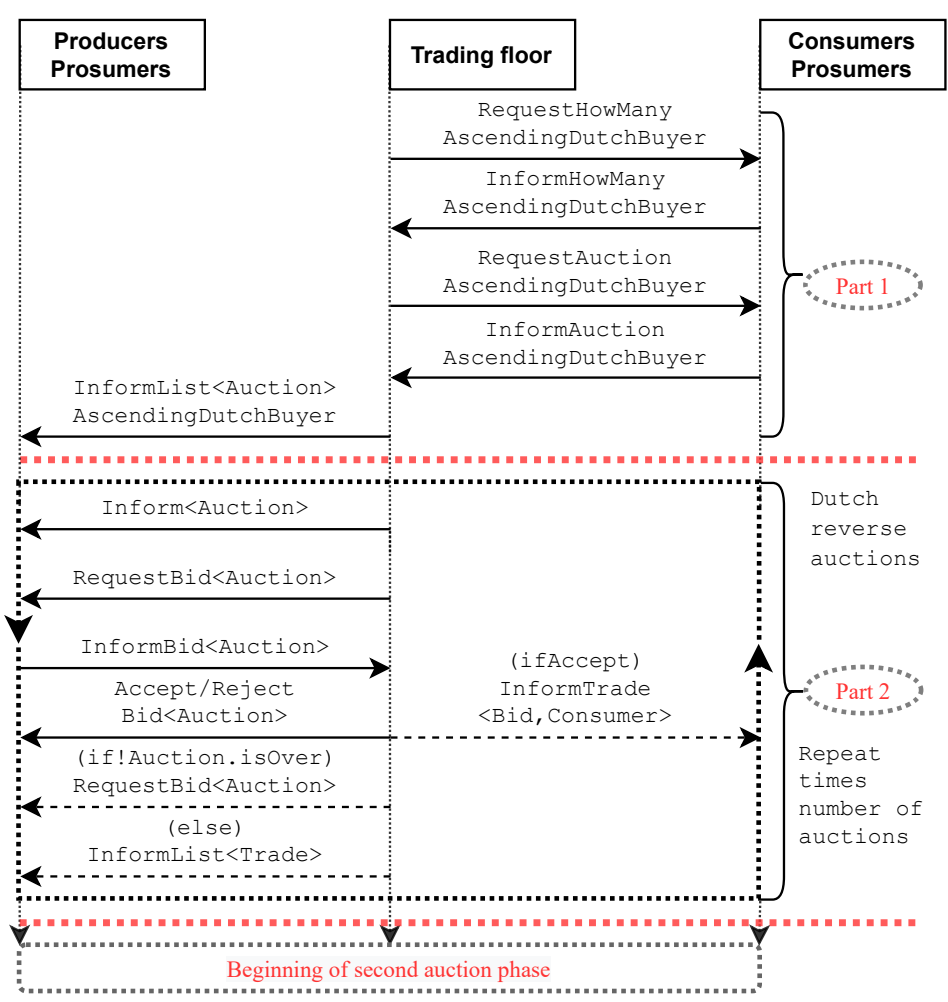

Fig. 3: First phase of auctions

\subsection{Prediction of aggregated supply and demand}

The goal of prediction protocol is to inform producers and consumers of what is feasible in the present timestep to let them place realistic bids and auctions.

The first step of the protocol is auction participants and the auction house agent identifying each other in their respective roles. Then, the auction house verifies who will participate in the upcoming auctions and asks them about their expected supply and demand in the next short-term trade. The agent will wait until everyone responds or enough time passes. Next, aggregated results are sent to all participants. Protocol ends with a message to each participant informing of that end. 


\subsection{Algorithm for the first auction phase}

All the auctions in the first phase are reverse closed dutch auctions. This phase contains two parts - establishing the number of auctions taking place and running the auctions. The phase starts by a query to consumers and prosumers on how many auctions they will submit (Fig. 3, part 1). The second part is running all the accepted auctions (Fig. 3, part 2). The phase starts by a query to consumers and prosumers on how many auctions they will submit (Fig. 3, part 1). Once buyers respond how many auctions they will submit, they are queried about the details of each auction - the desired quantity, budget and the initial price per ton of steam. Trading floor collects the submitted auctions. At the end of part one the submitted auctions are sorted and broadcasted.

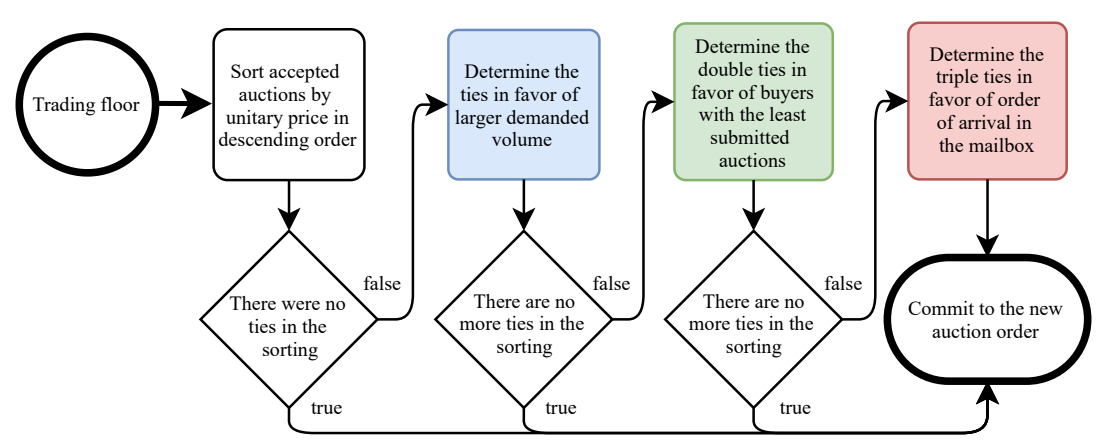

Fig. 4: Auction sorting algorithm

Sorting reverse Dutch auctions Fig. 4 presents the auction sorting algorithm. The first auctions are the ones with the highest unitary price of steam. Secondary sorting criterion is the purchase volume. Tertiary criterion is the number of auctions an agent has submitted - the lower the number, the higher the priority. If the previous criteria were insufficient to sort the auctions, the time of auction submission is considered. The chosen sorting order is dictated by several factors - the system consists of several players who may vary greatly in terms of size of their demand, which would give a natural advantage to larger buyers. Large share of control over demand would normally mean that steam producers focus on addressing their demand. Smaller buyers can compete with large vendors in terms of auction priority by raising base prices in auctions. The large vendors still have the advantage in the secondary order priority. The tertiary sorting criterion is aimed at limiting the possibility of scheming by the large vendors who submit a large amount of small auctions at various price points with the goal of gaining priority over small vendors while keeping the weighted average price across the auctions lower.

Accepting bids for every reverse Dutch auction Once all the auctions are in and sorted, the list of auctions is announced to the participants and producers as well as 


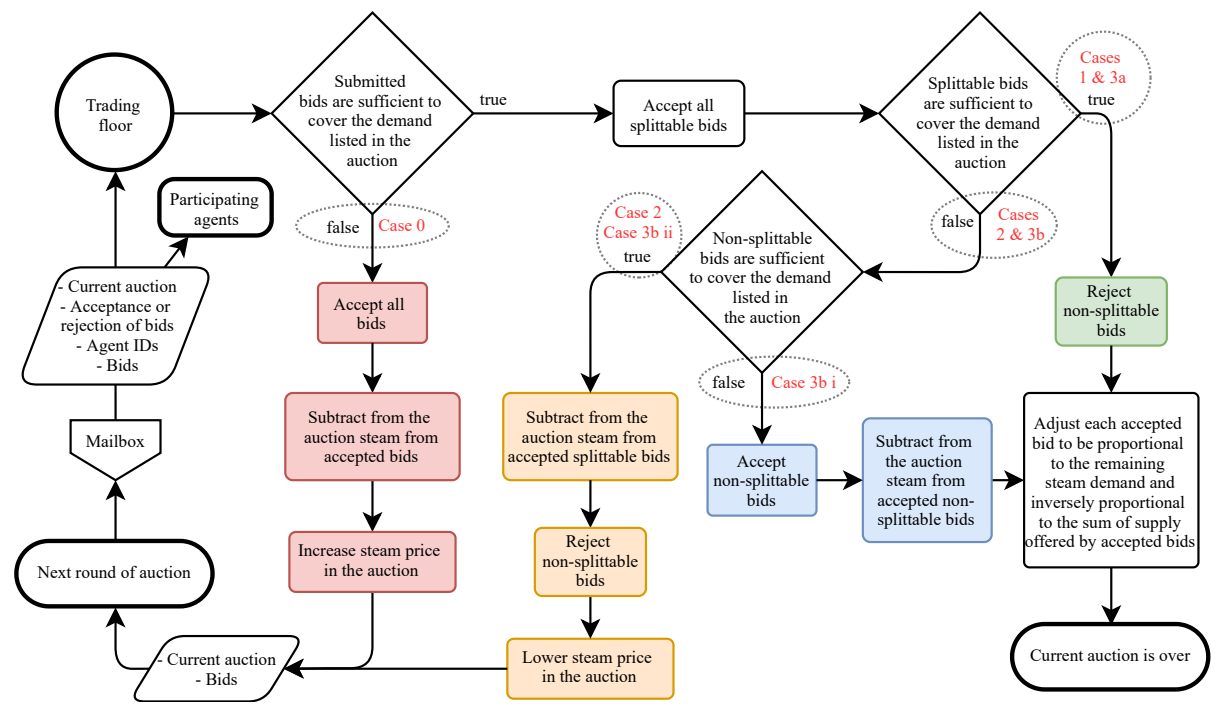

Fig. 5: Flowchart of one round of bid acceptance algorithm - starts at Trading Floor. Case 0: all incoming bids are accepted as the sum of offered volume does not cover the demand. Case 1: all bids are accepted but steam sold by each producer may be adjusted to meet its weighted average. Case 2: no steam is sold and the next round starts with a higher price. Case 3: some bids may be rejected. Red and orange paths lead to the next round. Green and blue steps conclude the auction.

prosumers are invited to bid in the first auction. The auction proceeds in the following fashion (Fig. 5): the trading floor sends the current unitary price to the bidders. They can respond how much volume they are willing to provide at the listed price and whether their bids are subject to partial acceptance. The bids are either accepted or rejected.

The rules of bet acceptance were created specifically for this trading system (see Fig. 5. Several cases are considered for each bid. When all submitted bids do not cover the demand of an auction, all bids are accepted, steam price is increased in the next round and the following cases do not apply (see Fig. 5, case 0).

1. All bidders accept incomplete orders :

The simplest situation is when all the bidders accept that their bid may be only partially accepted. In that case, the purchase volume accepted for each bidder is calculated as a weighted average (see equation 1 and Fig. 5, case 1)

2. All bidders refuse incomplete orders :

No steam is sold and the auction is reiterated with a lower price (Fig. 5, case 2)

3. Some bidders accept incomplete orders :

(a) Bids which accept split are sufficient to fulfil the orders :

In this case the resolution is the same as in case a with the exception that all the bids which do not permit splitting are rejected

(b) Bids which accept split are not sufficient to fulfil the orders (Fig. 5, case 3a)

i. Bids which cannot be split are insufficient to fulfil the demand:

All the non-splittable bids are accepted and the remaining demand volume will be split between splittable bids according to equation 1 (case $3 \mathrm{~b}$ i) 
ii. Bids which cannot be split are sufficient to fulfil the demand:

All the non-splittable bids are rejected and the remaining volume will be offered at a lower price (Fig. 5, case $3 \mathrm{~b}$ ii)

Equation 1 defines the weighted average of accepted volume for agent $A_{x}$ in case of partial bid acceptance.

$$
\operatorname{Accepted}\left(A_{x}\right)=\operatorname{Demand} \times \frac{\operatorname{Proposed}\left(A_{x}\right)}{\sum_{i=1}^{n} \operatorname{Proposed}\left(A_{i}\right)}
$$

The following examples illustrate how bid sorting would proceed when auction lists demand for volume $v_{1}$ at price $p_{1}$.

Example 1 All participants submit bids equal to $v_{1}$ and $p_{1}$ and make them splittable. In this case, all bids are accepted at $p_{1}$ but volume accepted from each bidder is equal to $v$ divided by number of bidders (Case 1 , green).

Example 2 One participant submits a bid equal to $v_{2}=\frac{v_{1}}{2}$ and makes it non-splittable, other participants submit bids equal to $v\left(p_{i}\right)$, where the sum of $v\left(p_{i}\right)$ is lower than $v_{1}$ but higher than $v_{2}$, and $p_{1}$ and make them splittable. All bids are accepted and the auction concludes. Bid of $v_{2}$ was accepted entirely whereas bids $v\left(p_{i}\right)$ were divided according to (equation 1 ), where demand is equal to $v_{1}-v_{2}$. (Case $3 \mathrm{~b} \mathrm{I}$, blue)

Example 3 All participants submit bids equal to $v\left(p_{i}\right)$ and $p_{1}$ and make them non-splittable. The sum of $v\left(p_{i}\right)$ is lower than $v_{1}$. All bids are accepted and the auction proceeds to next phase, with volume $v_{1}$ minus the sum of $v\left(p_{i}\right)$ and price $p_{2}>p_{1}$ (Case 0 , red).

The first phase of auctions is the main mechanism of steam trading in the system and aims at establishing fair market prices for both buyers an sellers while taking into account their limitations, such as flexibility and total size of their demand or supply.

\subsection{Algorithm for the second auction phase and delivery protocol}

The second auction phase is a descending dutch auction, starting at the lowest steam price in the first auction phase. This step is necessary to force sale of already produced steam at any price. Steam sellers are discouraged to sell steam through this auction as the price is now equal to the lowest established price in the previous auction phase.

First, sellers are asked for steam remaining after the first auction phase. The data is collected and, if there is any remaining steam, announced as a classic Dutch auction to buyers (Fig. 6, part 1). The auction starts at the lowest price steam was sold for in the first auction phase and the rules for bid acceptance are similar to those in Fig. 5 except only partial bets are allowed and price for remaining steam in the next round strictly goes down in increments until it reaches zero (Fig. 6, part 2). Once the steam is sold, lists of accepted trades are sent to respective buyers and sellers (Fig. 6, part 3). This concludes the protocol for short-term auctions and begins the delivery protocol.

The purpose of delivery protocol is twofold - bookkeeping and calculating the physical state of the steam network each timestep. The bookkeeping includes accounting for all established trades relevant to the timestep - both long-term and short-term. 


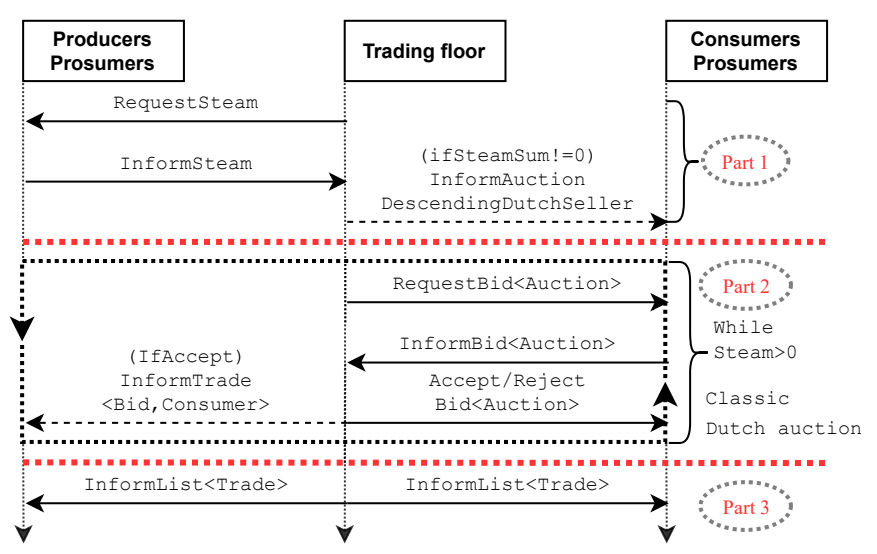

Fig. 6: Second phase of auctions

\section{Implementation and illustrative scenario}

The system is written in Java using JADE framework. Physical models of boilers and steam transport are simulated in MATLAB and integrated in Java. A special class, AugmentedAgent, was created to encompass basic functionalities needed by all agents in the system - address books, internal time tracking, role assignment. Its children are specific implementations for different technologies - boilers, energy storage, chemical plants, turbines, trading floors and broadcasters. Ontologies, protocols and other basic utility functions are used as decorators for instances of AugmentedAgent (see Fig. 7).

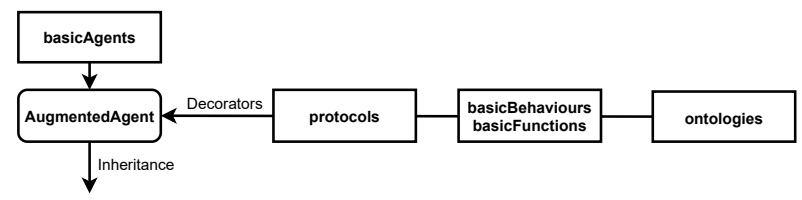

Fig. 7: Overview of code structure

Our platform allows us to model physical systems (boilers, heat storage,..) using MATLAB components and market participants through decision-making algorithms. The agents can generate demand curves, assess technical feasibility of meeting demand, production cost, $\mathrm{CO} 2$ emissions, long-term impact of possible decisions, implement strategy for efficient or optimal operation. For the end-users, the two major advantages of using a multi-agent approach are its flexibility and its privacy capabilities.

Data for simulations comes from real sources - historical data on fuel and electricity pricing from the European Energy Agency, prognoses of carbon emission certificates, performance tables of real boilers and High Temperature Gas-cooled Reactor (HTGR) designs. Real demand curves of chemical plants provided by our industrial partners will be used as a basis for simulating testing scenarios. We implemented several ontologies 
that are referred in our protocols. TradingOntology is common to all auction types, prediction and delivery protocols. SubscriptionOntology is used exclusively to update agents with current resource pricing and make questions regarding past pricing. The agents do not know future pricing in advance, with the exception of ExternalAgent.

The initial scenario consists of a HTGR, a gas boiler, a chemical plant and a turbine (Fig. 8). It is a very basic setup capable of both baseload and load following.

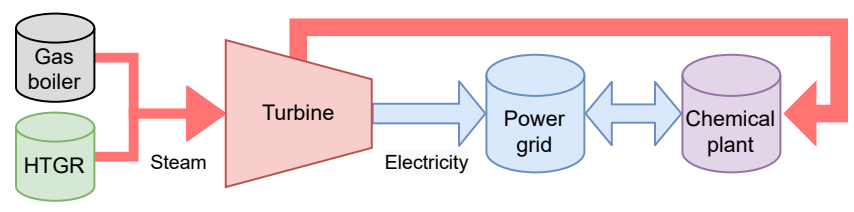

Fig. 8: Visualization of a physical layer of the illustrative scenario

HTGR is simulated with table values of max ramp-up and down speeds as well as a simple Xenon decay model which will reduce the max speeds. Gas boiler is a simpler model, based around fuel efficiency curve and max ramp-up and down speeds. Both units is of equal thermal power - $100 \mathrm{MW}$. They are connected to a back-pressure turbine, presumed to handle the load. Its isentropic efficiency is considered constant for every load factor. In the basic scenario, only high-pressure steam is sold, with lower pressures being converted to electricity. Electricity sales are handled by the grid agent at market prices at the time of trade as direct sales. The purpose of the scenario is to test the functionality of the system, agent responses in steadily changing conditions and efficiency of multi-stage short-term auction mechanisms.

In the Steady-state scenario, both the load factor of the chemical plant and pricing of all resources is constant. Several prices are chosen to evaluate common-sense decisions with high and low fuel and emission prices as well as several levels of load of the chemical plant. The expected result is a converging steam price in each case. Timeframe of this scenario is extended until the price of steam converges to a steady state or one year. Timestep for short-term auctions is set to one minute.

Different load and pricing scenarios implement three variations: a) variable load factor with constant pricing, b) constant load factor with variable pricing and c) variable load factor and pricing. They investigate the extent to which a decision-making based on production cost as a sole criterion for profitability can ensure continued supply.

\section{Related work}

Decarbonisation of industrial heat production is among most challenging tasks in the field. The main reason is the dependence of the industry on reliable and flexible supply of high-temperature heat, mostly in the form of steam. While combustion of fossil fuels yields high temperatures easily, potential emission-free sources have great difficulty providing it. HGTRs are a solution to the problem but are burdened with high capital 
cost, lower flexibility and both legal and political hurdles $[6,7]$. To maintain economic viability, industrial complexes should consider new approaches to energy management.

The challenge of decarbonizing industrial complexes with alternative management methods and introduction of HGTRs relates to a more general problem of managing distributed energy production. Several algorithms and methods have been proposed to achieve it [8]. There are three main approaches to simulating systems operations Discrete Event, System Dynamics and Agent-Based Simulation [9, 10]. Discrete Event Simulations are highly procedural. System Dynamic methods focus on modelling state dynamics with equations defining rates of change, it is analogous to physics simulations. Agent modelling is based on autonomous actors interacting with each other within a set of rules. They can have complex decision-making algorithms and can exhibit self-coordination [11] while ensuring the privacy of the various actors they represent. The two properties are among the key factors for selecting the agent modelling approach.

Multi-agent approach has been successfully applied to energy systems. MAS is used in many smart grids applications [12,13], where the challenge of load balancing with intermittent energy production is common. Microgrids and District heating are two examples where MAS have been successfully deployed [11, 14, 15]. The examples share common traits with energy systems of industrial complexes. The differences are the covariance between process heat and electricity, the constrained number of actors in the system combined with the paramount importance of QoS and co-dependence between consumers and producers.

On a spectrum of classification of MAS, our system is a case of decentralized control system with overall objectives. The agents are heterogeneous and their hierarchy is holonic - outside of special agents responsible for coordination of actions, the only commitments between agents are ongoing energy contracts $[14,16]$. One of the related multi-agent tools is GRENAD [17]. It uses a similar division of roles into distribution, production and consumption as our system. It is not open-source, though, and is designed for smart grids rather than production facilities.

Market-based approach to meeting supply and demand with MAS is a well-known and promising approach [18]. Typical approach in energy markets is based around establishing market clearing prices, where all energy is sold at a set price and every producer margins are different, or sealed-bid auctions, where either buyers or sellers make "take it or leave it" offers. Other approaches use indicators of importance to allocate optimal energy production and distribution [16, 18]. However, chemical plants cannot properly schedule their production in face of large price and supply volatility, leading to overbuilt energy supply systems in real life. The nature of our system has led us to develop our own method of allocating resources through adapting market mechanisms. Our long and short-term auctioning approach combines traits of both approaches and minimizes the risk of excess energy production and should enable fair profit margins to every actor, if one's decision making is sound.

Unlike energy grid or stock market, industrial complexes have few actors. Combined with volatile demand, it leads to low resilience compared to a grid or an entire market. Additionally, the allocated goods - heat and electricity - have more complex cost structures compared to electricity on the grid, as the chemical plants are fully dependent on heat supplied from boilers. The setup creates a coopetition - energy producers need to 
remain profitable themselves while ensuring long-term profitability of chemical plants. Power plants in the complexes were not designed for efficient electricity production and will not sustain themselves without heat consumers. Both parties need to make fair trades to ensure mutual success. One of the promising approaches to solve the coopetition problem using MAS are cooperative VPPs, which would lead to fewer but more reliable auction participants and could take better advantages of potential synergies among technologies [19].

One of the difficulties with validating the systems is the environment - for both economic and safety reasons, the tests cannot be easily carried out on real energy systems. It was faced in multiple cases for smart grids. Obtaining both realistic data and parameters to model agents is challenging [20]. Special tools were created as testbeds for MAS and for monitoring their performance. A potential step to test its real-life performance is a test loop available for research at INL [21]. MAS studies of energy systems have based their validation on parameters and historical data of real-life systems and we will follow this approach [22].

\section{Conclusion and Perspectives}

This paper proposes a decentralized control system of energy in chemical complexes with multi-stage auction mechanisms for short-term steam trading to optimize fuel consumption, increase profits and lower $\mathrm{CO}_{2}$ emissions. The proposed auction mechanism sells all produced steam every timestep while meeting the demand and including all parties in the negotiations fairly. It combines classic and reverse dutch auctions with custom rules. This proposal is a first step towards an industrially deployable steam distribution system with multiple producers and consumers in the context of a HTR used as steam producer in chemical complexes and Hybrid Energy Systems (HES).

To evaluate our work, we developed a simulator standing on real physical models and datasets. It will be tested with simple scenario parameters - steady-state or variable load and demand, based on historical data. The next step involves adding long term trade and increased the complexity of the system, with new and more advanced physical agents and long-term scenarios. Once all elements are introduced, scenarios with malfunctions, outages and system changes will be assessed.

\section{References}

1. B. B. Botros and J. G. Brisson, "Targeting the optimum steam system for power generation with increased flexibility in the steam power island design," Energy, vol. 36, no. 8, pp. 4625-4632, 2011.

2. Haverhill north coke copany and Sunoco inc. (R\&M), "Steam supply and purchase agreement," 2010.

3. Kumana Associates, "How to calculate the true cost of steam," U.S. Dept. of Energy, 2003.

4. Y. Shoham and K. Leyton-Brown, "Multiagent systems: Algorithmic, Game-Theoretic, and logical foundations," Multiagent Systems: Algorithmic, Game-Theoretic, and Logical Foundations, vol. 9780521899, pp. 1-483, 2008.

5. J. Buchanan, S. Gjerstad, and D. Porter, "Information effects in uniform price multi-unit dutch auctions," Southern Economic Journal, vol. 83, no. 1, pp. 126-145, 2016. 
6. A. Bredimas, K. Kugeler, and M. A. Fütterer, "Strengths, weaknesses, opportunities and threats for HTR deployment in Europe," Nuclear Engineering and Design, vol. 271, pp. 193-200, 2014.

7. M. F. Ruth, O. R. Zinaman, M. Antkowiak, R. D. Boardman, R. S. Cherry, and M. D. Bazilian, "Nuclear-renewable hybrid energy systems: Opportunities, interconnections, and needs," Energy Conversion and Management, vol. 78, pp. 684-694, 2014.

8. A. L. Kulasekera, R. A. Gopura, K. T. Hemapala, and N. Perera, "A review on multi-agent systems in microgrid applications," 2011 IEEE PES International Conference on Innovative Smart Grid Technologies-India, ISGT India 2011, no. December, pp. 173-177, 2011.

9. R. Maidstone, "Discrete Event Simulation, System Dynamics and Agent Based Simulation: Discussion and Comparison," System, pp. 1-6, 2012.

10. W. C. Baldwin, B. Sauser, and R. Cloutier, "Simulation approaches for system of systems: Events-based versus agent based modeling," Procedia Computer Science, vol. 44, no. C, pp. 363-372, 2015.

11. A. Gonzalez-Briones, F. De La Prieta, M. S. Mohamad, S. Omatu, and J. M. Corchado, "Multi-agent systems applications in energy optimization problems: A state-of-the-art review," Energies, vol. 11, no. 8, pp. 1-28, 2018.

12. J. Gil-Quijano, "Post-doctorate: Distributed optimal scheduling of energy resources. Application to district heating.," 2013.

13. F. Dötsch, J. Denzinger, H. Kasinger, and B. Bauer, "Decentralized real-time control of water distribution networks using self-organizing multi-agent systems," Proceedings - 2010 4th IEEE International Conference on Self-Adaptive and Self-Organizing Systems, SASO 2010, no. September, pp. 223-232, 2010.

14. K. Ghribi, S. Sevestre, Z. Guessoum, J. Gil-Quijano, D. Malouche, and A. Youssef, "A survey on multi-agent management approaches in the context of intelligent energy systems," 2014 International Conference on Electrical Sciences and Technologies in Maghreb, CISTEM 2014, no. January 2015, 2014.

15. M. A. Souissi, K. Bensaid, and R. Ellaia, "Multi-agent modeling and simulation of a stock market," Investment Management and Financial Innovations, vol. 15, pp. 123-134, 2018.

16. J. Kamphuis, C. J. Kok, and I. G. Warmer, "PowerMatcher: Multiagent Control in the Electricity Infrastructure," Energy research Center of the Netherlands (ECN), vol. 32, no. 18, pp. 151-155, 2010.

17. S. Ductor, J. J. Gil-Quijano, N. Stefanovitch, and P. R. Mele, "GRENAD, a modular and generic smart-grid framework," Proceedings of the 2015 Federated Conference on Computer Science and Information Systems, FedCSIS 2015, vol. 5, pp. 1781-1792, 2015.

18. F. Ygge and H. Akkermans, "Power Load Management as a Computational Market," Proceedings of the Second International Conference on Multi-Agent Systems (ICMAS, no. March, pp. 1-14, 1996.

19. G. Chalkiadakis, V. Robu, R. Kota, A. Rogers, and N. R. Jennings, "Cooperatives of distributed energy resources for efficient virtual power plants," 10th International Conference on Autonomous Agents and Multiagent Systems 2011, AAMAS 2011, vol. 2 , no. May 2014, pp. 737-744, 2011.

20. Y. Atif, Y. Jiang, B. Lindström, J. Ding, M. Jeusfeld, S. Andler, E. Nero, C. Brax, and D. Haglund, "ELVIRA: Multi-agent Systems for Power Grid Monitoring," pp. 1-16, 2018.

21. J. E. O'Brien, P. Sabharwall, and S. Yoon, "A multi-purpose thermal hydraulic test facility for support of advanced reactor technologies," Transactions of the American Nuclear Society, vol. 111, pp. 1639-1642, 2014.

22. H. Ren, W. Zhou, K. Nakagami, W. Gao, and Q. Wu, "Multi-objective optimization for the operation of distributed energy systems considering economic and environmental aspects," Applied Energy, vol. 87, no. 12, pp. 3642-3651, 2010. 